\title{
Phenotypic Characterization of Recent Clonal Lineages of Phytophthora infestans in the United States
}

\author{
G. Danies, I. M. Small, K. Myers, R. Childers, and W. E. Fry, Department of Plant Pathology, Cornell University, Ithaca, NY 14853
}

\begin{abstract}
Danies, G., Small, I. M., Myers, K., Childers, R., and Fry, W. E. 2013. Phenotypic characterization of recent clonal lineages of Phytophthora infestans in the United States. Plant Dis. 97:873-881.

Phytophthora infestans, the causal agent of late blight disease, has been reported in the United States and Canada since the mid-nineteenth century. Due to the lack of or very limited sexual reproduction, the populations of $P$. infestans in the United States are primarily reproducing asexually and, thus, show a simple genetic structure. The emergence of new clonal lineages of $P$. infestans (US-22, US-23, and US24) responsible for the late blight epidemics in the northeastern region of the United States in the summers of 2009 and 2010 stimulated an investigation into phenotypic traits associated with these genotypes. Mating type, differences in sensitivity to mefenoxam, differences in pathogenicity on potato and tomato, and differences in rate of germina-

Both A1 and A2 mating types were detected. Lineages US-22, US-23, and US-24 were generally sensitive to mefenoxam while US-8 was resistant. US- 8 and US- 24 were primarily pathogenic on potato while US-22 and US-23 were pathogenic on both potato and tomato. Indirect germination was favored at lower temperatures $\left(5\right.$ and $\left.10^{\circ} \mathrm{C}\right)$ whereas direct germination, though uncommon, was favored at higher temperatures $\left(20\right.$ and $\left.25^{\circ} \mathrm{C}\right)$. Sporangia of US-24 released zoospores more rapidly than did sporangia of US-22 and US-23. The association of characteristic phenotypic traits with genotype enables the prediction of phenotypic traits from rapid genotypic analyses for improved disease management.
\end{abstract} tion were studied for clonal lineages US-8, US-22, US-23, and US-24.
Phytophthora infestans, the causal agent of late blight disease, has been reported in the United States since the 1840s (26). Since its introduction, it is hypothesized that the pathogen population has been primarily reproducing clonally, and new clonal lineages have emerged mainly by mitotic recombination, mutation, or migration events (5). Due to the simple population structure of $P$. infestans in the United States, it has been possible to group individuals into clonal lineages based on neutral markers such as mating type, mitochondrial haplotype, nuclear DNA fingerprint patterns, and allozyme genotype (5). More recently, this approach has been complemented with the use of microsatellite markers $(22,24)$.

Isolates within lineages are recognized to be more similar to each other than to isolates in a different clonal lineage for characteristics such as metalaxyl or mefenoxam resistance and host preference $(8,9,15,17,20)$. Several studies have characterized previously dominant clonal lineages and demonstrated the existence of considerable, predictable variation among different clonal lineages in epidemiologically important phenotypic traits. Some examples include phenylamide fungicide resistance in clonal lineages US-7 and US-8 (7) and the fact that US-8 is pathogenic mainly on potato whereas US-7, US-11, and US-17 are pathogenic on both potato and tomato $(7,17)$.

Environmental variables such as temperature and relative humidity have a significant impact on the development of late blight. Early studies conducted by Melhus (19) and Crosier (2) indicated

Corresponding author: W. Fry, E-mail: wef1@ cornell.edu

* The $\boldsymbol{e}$-Xtra logo stands for "electronic extra" and indicates that a supplementary table is available online.

Accepted for publication 7 January 2013.

http://dx.doi.org/10.1094/PDIS-07-12-0682-RE

(c) 2013 The American Phytopathological Society that indirect germination was predominant at temperatures below $20^{\circ} \mathrm{C}$. In a study by Mizubuti et al. (21), differential effects of temperature on sporangia germination were reported; the optimal temperature for indirect germination was lower for lineages US-7 and US-8 than it was for US-1. Clonal lineages US-7 and US-8 germinated indirectly at temperatures below $15^{\circ} \mathrm{C}$. The differential effects of temperature on sporangia germination may differentially influence the efficacy of sporangia to initiate infection and, therefore, are important for disease epidemiology and disease management.

The reemergence of late blight in northeastern United States and Canada in the summers of 2009 and 2010 revealed the existence of new clonal lineages of $P$. infestans (12). Both potato and tomato were infected by the new clonal lineages. Knowledge regarding the sensitivity of the new genotypes to the fungicide mefenoxam, their host preference, and other epidemiological characteristics could provide information important to disease management decisions. The objective of this study was to investigate epidemiologically important phenotypic characteristics of the recently emerged clonal lineages in the United States and Canada. Sensitivity to the systemic fungicide mefenoxam, pathogenicity on potato and tomato, and the effect of temperature on sporangium germination were investigated for clonal lineages US-8, US-22, US-23, and US-24.

\section{Materials and Methods}

Isolates and isolation. Isolates were obtained from throughout the United States and from select provinces in Canada during 2009, 2010, and 2011. Sampling of $P$. infestans isolates is limited to those places where the pathogen occurs and cannot be predicted from one season to another. For this reason a predetermined sampling strategy could not be employed. Isolates collected for this study were those reported by extension personnel and plant disease diagnostic labs in our network. Isolates were maintained on pea agar (13), rye B agar (1), and tomato or potato leaflets (depending on the isolate) at $15^{\circ} \mathrm{C}$. When the source of the isolates was sporulating lesions, a small block of media (rye B and pea agar) with 
antibiotics (ampicillin at $100 \mu \mathrm{g} \mathrm{ml}^{-1}$, rifampicin at $125 \mu \mathrm{g} \mathrm{m} \mathrm{m}^{-1}$, and pentachloronitrobenzene at $25 \mu \mathrm{g} \mathrm{ml}^{-1}$ ), was placed in contact with sporangia, which was then transferred to petri plates of agar medium. From the plates, colonies were selected and transferred again onto rye $\mathrm{B}$ and pea agar media.

Mating type. Mating type was determined by pairing an unknown isolate with a known isolate of $P$. infestans, either A1 mating type (US970001, US-17 genotype) or A2 mating type (US040009, US-8 genotype), on rye B or pea agar media. Petri plates were kept at $20^{\circ} \mathrm{C}$ for 10 to 14 days. The hyphal interface of the two colonies was investigated microscopically using $\times 125$ magnification. Isolates that formed oospores at the interface with the known A1 isolate were designated A2 and those that formed oospores with the known A2 isolate were designated A1. The known isolates (A1 and A2) were paired as positive controls, while negative controls consisted of pairing the known isolates with themselves (same mating type).

Mitochondrial haplotype. Mitochondrial DNA haplotype was determined using polymerase chain reaction (PCR) restriction fragment length polymorphism (RFLP) analysis, as described by Griffith et al. (11), with two primer pairs (F1-R1 and F2-R2). Reference US-1 and US-8 isolates were included as positive controls.

Glucose-6-phosphate isomerase. Mycelia or sporangia obtained from cultures grown on rye B or pea agar or from infected leaflets were used to determine glucose-6-phosphate isomerase (GPI) allozyme genotypes. Analyses were carried out using cellulose acetate electrophoresis as described by Goodwin et al. (6). One or more reference isolates representing US-1 (SA960008), US-8 (US040009), or US-17 (US970001) were included in all GPI analyses.

DNA extraction and RFLP analysis with probe RG-57. DNA extractions and subsequent RFLP analysis with the RG-57 DNA probe were performed using a method modified from Goodwin et al. (10). Southern blot analysis was conducted using the Amersham gene images AlkPhos direct labeling and detection system (GE Healthcare), according to the manufacturer's instructions. The US1 (SA960008) reference isolate was used in RG-57 analyses. Presence or absence of all fingerprint fragments was scored visually.

Multiplex microsatellite marker analysis. Simple sequence repeats (SSRs) were analyzed for all isolates, using protocols developed previously (16) and described in the Protocol section, March 2008, by the Eucablight Network (www.eucablight.org). Three separate multiplex reactions were conducted using three panels of primers (Table 1). Master mixes were prepared. Amounts per single reaction are shown in Table 2. PCR amplification was conducted with a standardized set of thermocycling conditions: initial activation step of $95^{\circ} \mathrm{C}$ for $10 \mathrm{~min}$; followed by 28 cycles of $95^{\circ} \mathrm{C}$ for $20 \mathrm{~s}, 58^{\circ} \mathrm{C}$ for $25 \mathrm{~s}$, and $72^{\circ} \mathrm{C}$ for $60 \mathrm{~s}$; and a final extension step of $72^{\circ} \mathrm{C}$ for $20 \mathrm{~min}$. Post-PCR processing was conducted by dispensing $9.5 \mu$ of size standards in high-deionized formamide mix, $0.05 \mu \mathrm{l}$ of Genescan-500 LIZ size standard (Applied Biosystems [ABI], PN 4322682) and $9.45 \mu \mathrm{l}$ of Hi-Di Formamide (ABI, PN 4311320), and $0.5 \mu \mathrm{l}$ each of the PCR products from panel 1-3 reactions into each well of a 96-well ABI 3730xl plate. PCR products were analyzed on an ABI 3730xl capillary system with POP-7 Polymer (ABI, PN 4335615). PCR amplicons were compared with a set of size standards and alleles were scored accordingly (16).

Table 2. Polymerase chain reaction (PCR) reactions for simple sequence repeat analysis for Phytophthora infestans isolates ${ }^{\mathrm{a}}$

\begin{tabular}{|c|c|c|c|}
\hline \multirow[b]{2}{*}{ Reagents } & \multicolumn{3}{|c|}{ Volume $(\mu \mathrm{l})$} \\
\hline & Plate 1 & Plate 2 & Plate 3 \\
\hline HPLC-grade water ${ }^{\mathrm{b}}$ & 7.95 & 6.79 & 8.375 \\
\hline $10 \times$ Immolase PCR buffer & 1.2 & 1.2 & 1.25 \\
\hline 10 mM dNTPs & 0.125 & 0.187 & 0.125 \\
\hline $50 \mathrm{mM} \mathrm{MgCl}_{2}$ & 0.375 & 0.42 & 0.375 \\
\hline \multicolumn{4}{|l|}{$10 \mu \mathrm{M}$ primers $^{\mathrm{c}}$} \\
\hline $\mathrm{Pi} 02$ & 0.125 & $\ldots$ & $\ldots$ \\
\hline Pi89 & 0.275 & $\ldots$ & $\ldots$ \\
\hline Pi4B & 0.475 & $\ldots$ & $\ldots$ \\
\hline PiG11 & $\ldots$ & 0.3 & $\ldots$ \\
\hline Pi04 & $\ldots$ & 0.125 & $\ldots$ \\
\hline Pi70 & $\ldots$ & 0.375 & $\ldots$ \\
\hline Pi56 & $\ldots$ & 0.3 & $\ldots$ \\
\hline Pi63 & $\ldots$ & 0.3 & \\
\hline Pi16 & $\ldots$ & $\ldots$ & 0.188 \\
\hline Pi33 & $\ldots$ & $\ldots$ & 0.1 \\
\hline D13 & $\ldots$ & $\ldots$ & 0.35 \\
\hline Immolase Taq $\left(5 \mu \mu^{-1}\right)$ & 0.1 & 0.1 & 0.1 \\
\hline Template DNA $\left(\approx 1 \mathrm{ng} \mu^{-1}\right)$ & 1 & 1 & 1 \\
\hline
\end{tabular}

${ }^{a}$ Multiplex PCR reactions were conducted using three panels of primers based on a protocol developed by the Eucablight Network (www. eucablight.org) as described in the Protocol section March 2008. Reagents used were from Bioline.

${ }^{\mathrm{b}} \mathrm{HPLC}=$ high-performance liquid chromatography.

${ }^{c}$ Forward and reverse primers.

Table 1. Phytophthora infestans microsatellite primer information

\begin{tabular}{|c|c|c|c|c|c|c|}
\hline Primer & Primer sequence & $\begin{array}{l}\text { Cooke allele (bp) } \\
\text { US lineages }(\text { bp })^{\mathrm{a}}\end{array}$ & Label & $\begin{array}{c}\text { Annealing } \\
\text { temperature }\left({ }^{\circ} \mathbf{C}\right)\end{array}$ & Repeat & Reference \\
\hline D13F & F: TGCCCCCTGCTCACTC & $106-166$ & 6-FAM & 56 & $(\mathrm{CT})_{27}$ & 16 \\
\hline D13RLong & R: GCTCGAATTCATTTTACAGACTTG & $106-147$ & $\ldots$ & $\ldots$ & & (modified) \\
\hline $\mathrm{Pi} 02 \mathrm{~F}$ & F: CAGCCTCCGTGCAAGA & $142-168$ & PET & 58 & $(\mathrm{TG})_{11}$ & 16 \\
\hline Pi02R & R: AAGGTGCGCGAAGACC & $154-166$ & $\ldots$ & & & $\ldots$ \\
\hline Pi4BFcap & F: AAAATAAAGCCTTTGGTTCA & $203-225$ & NED & 58 & $(\mathrm{TC})_{34}$ & 14 \\
\hline Pi4BR & R: GCAAGCGAGGTTTGTAGATT & $213-226$ & $\ldots$ & $\ldots$ & & $\ldots$ \\
\hline PiG11Fcap & F: TGCTATTTATCAAGCGTGGG & $138-212$ & VIC & 56 or 58 & $(\mathrm{TC})_{26}$ & 14 \\
\hline PiG11R & R: TACAATCTGCAGCCGTAAGA & $127-159$ & $\ldots$ & ... & $\ldots$ & $\ldots$ \\
\hline Pi56Fcap & F: AACTATCTATCGGCGTGCAT & $252-255$ & PET & 58 & $(\mathrm{AT})_{10}$ & 16 \\
\hline Pi56RLong & R: CAGGCCGCAATTGTAAGA & $255-257$ & $\ldots$ & & $\ldots$ & $\ldots$ \\
\hline Pi04Fcap & F: AGCGGCTTACCGATGG & $160-172$ & NED & 58 & $(\mathrm{GT})_{6}$ & 16 \\
\hline Pi04R & R: CAGCGGCTGTTTCGAC & $166-170$ & $\ldots$ & & $\ldots$ & $\ldots$ \\
\hline Pi63F & F: ATGACGAAGATGAAAGTGAGG & $272-281$ & 6-FAM & 58 & $(\mathrm{GAG})_{8}$ & 16 \\
\hline Pi63Rlong & R: CGTATTTTCCTGTTTATCTAACACC & $269-279$ & $\ldots$ & & & $\ldots$ \\
\hline Pi33Fcap & F: TGCCGACGACAAGGAA & $203-209$ & VIC & 58 & $(\mathrm{CAG})_{5}$ & 16 \\
\hline Pi33R & R: CGGTCTGCTGCTGCTC & $203-206$ & $\ldots$ & $\ldots$ & $\ldots$ & $\ldots$ \\
\hline Pi16Fcap & F: CACAGCACGCGGAATC & $173-177$ & NED & 58 & $(\mathrm{GA})_{7}$ & 16 \\
\hline Pi16R & R: ACGCCGAGTGTCCTGA & $173-177$ & $\ldots$ & $\ldots$ & $\ldots$ & $\ldots$ \\
\hline $\mathrm{Pi} 70 \mathrm{~F}$ & F: ATGAAAATACGTCAATGCTCG & 189-195 & 6-FAM & 58 & $(\mathrm{AAG})_{8}$ & 16 \\
\hline Pi70R & R: CGTTGGATATTTCTATTTCTTCG & $187-193$ & $\ldots$ & $\ldots$ & $\ldots$ & $\ldots$ \\
\hline Pi89Fcap & F: GAGAACGCACAATGTAAGGC & $177-211$ & 6-FAM & 58 & $(\mathrm{AT})_{9}$ & 16 \\
\hline Pi89R & R: ACATAAATACACGCTGAACGG & $177-181$ & $\ldots$ & $\ldots$ & $\ldots$ & $\ldots$ \\
\hline
\end{tabular}

a Size ranges. 
Mefenoxam sensitivity assay. Mefenoxam sensitivity of isolates was assessed as described previously by Therrien et al. (27), except that mefenoxam was used in place of metalaxyl. Isolates were grown on V8 agar or pea agar amended with Ridomil Gold $\mathrm{EC}$, of which $49 \%$ was the active ingredient (mefenoxam) (Syngenta), such that the final concentrations of the active ingredient were 0,5 , or $100 \mu \mathrm{g} \mathrm{ml}^{-1}$. Mycelial plugs ( $8 \mathrm{~mm}$ in diameter) were obtained from actively growing cultures, transferred to the test plates, and incubated for approximately 10 to 12 days, or until growth on the control mefenoxam plate $\left(0 \mu \mathrm{g} \mathrm{ml}^{-1}\right)$ was approximately $80 \%$ of the diameter of the petri plate. Assessment of mefenoxam sensitivity was determined on the basis of radial growth of cultures grown on plates amended with mefenoxam (5 or $100 \mu \mathrm{g} \mathrm{ml}^{-1}$ ) compared with nonamended controls. Growth on mefenoxam-amended plates, 5 and $100 \mu \mathrm{g} \mathrm{ml}^{-1}$, was represented as a proportion of the growth on the nonamended control plates.

Effects of mefenoxam concentration, lineage, and year of collection on colony growth were analyzed using JMP 9.0.2 (SAS Institute). Standard least square analysis was used, where replications were considered random terms, while mefenoxam concentration, lineage, and year of collection were considered as fixed effects. To determine whether means of colony growth on mefenoxamamended plates, for each lineage, differed between years, a TukeyKramer HSD (honestly significant difference) test with $\alpha=0.05$ was performed.

Inoculum production. A sporangial suspension was used for inoculation of potato and tomato leaflets. Sporangia were washed from sporulating lesions on tomato or potato leaflets, which had been maintained in water-agar moist chambers at $15^{\circ} \mathrm{C}$ with a $16-\mathrm{h}$ light period for 8 days prior to inoculation. The sporangial suspension was adjusted to 4,000 sporangia $\mathrm{ml}^{-1}$ using a hemocytometer and maintained at $4^{\circ} \mathrm{C}$ for $2 \mathrm{~h}$ before being applied, by pipetting 20 $\mu \mathrm{l}$ on a leaflet. Leaflets were obtained from 4- to 5-week-old ' $\mathrm{Yu}$ kon Gold' potato and 'Rutgers' tomato plants grown under greenhouse conditions.

Potato and tomato pathogenicity. In order to determine differences in host preference for isolates, each isolate was inoculated onto both potato Yukon Gold and tomato Rutgers leaflets. In all, 8 US-8, 34 US-22, 7 US-23, and 9 US-24 isolates were used for this study (Supplementary Table S1). Plants were grown in the greenhouse (approximately $25^{\circ} \mathrm{C}$ daytime and $20^{\circ} \mathrm{C}$ nighttime) and, when 4 to 5 weeks old, recently matured leaflets were harvested. Inoculations were carried out in 150 -mm petri plates containing 75 $\mathrm{ml}$ of water agar $(1.5 \%)$ in the smaller half, which served as the lid (top). Leaflets were placed (abaxial side up) on the base of the moist chamber. Each moist chamber contained five potato or five tomato leaflets, abaxial side up. All five leaflets were inoculated with $20 \mu \mathrm{l}$ of a sporangial suspension (described above) of the same isolate, deposited on one side of the main vein of the leaflet. After the leaflets were inoculated, the petri plate was sealed with parafilm and incubated at $15^{\circ} \mathrm{C}$ with a 16 -h light period. The experiment was conducted at least twice for each isolate.

Lesion size and number of sporangia per lesion were measured at 6 days after inoculation. Lesion areas were estimated by taking two perpendicular measurements (length and width) starting from the widest diameter, using a ruler. Subsequently, the number of sporangia produced on each lesion was determined. Individual lesions were excised and placed into 14-ml disposable polypropylene culture tubes with $3 \mathrm{ml}$ of preservative solution ( $0.04 \mathrm{M}$ copper sulfate, $0.2 \mathrm{M}$ sodium acetate, and acetic acid, $\mathrm{pH}$ 5.4) (25). The tubes were then vortexed for $10 \mathrm{~s}$ to dislodge and suspend sporangia, and aliquots were counted with a hemocytometer. Hemocytometer counts were repeated at least twice. The total number of sporangia per lesion was then calculated by averaging all the independent counts. The assay was conducted at a standardized temperature of $15^{\circ} \mathrm{C}$. Lesion area and sporulation were compared between potato and tomato for each clonal lineage separately. The statistical significance of differences in mean lesion size and sporangia production between hosts, for each clonal lineage, was determined using a Student's $t$ test with $\alpha=0.05$.
Temperature effect on sporangium germination. The effect of temperature on sporangium germination was assessed. Three 400$\mu \mathrm{l}$ droplets of $1.5 \%$ water agar were dispensed into three independent circular silicone-molds placed on top of a glass microscope slide. A $20-\mu \mathrm{l}$ droplet of a suspension consisting of 4,000 sporangia $\mathrm{ml}^{-1}$ was deposited on each water agar droplet. Three isolates for each lineage (US-8, US-22, US-23, and US-24) were used for this study (Supplementary Table S1). Inoculated slides were placed in incubators at $10,15,20$, and $25^{\circ} \mathrm{C}$ with no light. After $16 \mathrm{~h}$, total germination (direct, indirect, and no-germination) was determined by microscopic observation of each water agar droplet. For each isolate, three replicates were conducted at each temperature, and the experiment was repeated at least twice. In a second experiment, germination was assessed at 5 and $10^{\circ} \mathrm{C}$.

Effects of temperature and lineage on germination (indirect or direct) were analyzed using JMP 9.0.2 (SAS Institute). Standard least square analysis was used, where replications were considered random terms and temperature and lineage were considered fixed effects. To determine whether mean germination differed between temperatures, for each lineage, a Tukey-Kramer HSD test with $\alpha=$ 0.05 was performed.

Effect of temperature on the rate of sporangium germination. Sporangia were observed at 30, 90, 120, 240, and $960 \mathrm{~min}$ after incubation at 15 and $4^{\circ} \mathrm{C}$. Inoculation was performed as described above. For the $15^{\circ} \mathrm{C}$ assay, a single slide with three independent repetitions (three circular water agar droplets that had each been inoculated with $20 \mu$ of the same sporangial suspension) was assessed for the five time points studied, for each isolate. Total germination (direct, indirect, and no-germination) was counted at the first 30-min assessment and, thereafter, only indirect germination (sporangia that had released their zoospores) was recorded. This was possible because slides could be incubated and assessed at $15^{\circ} \mathrm{C}$ for the duration of the assay. For the $4{ }^{\circ} \mathrm{C}$ assay, independent measurements of total germination were carried out for each respective time point. That is, a different slide with three circular water agar droplets that had each been inoculated with $20 \mu$ of the same sporangial suspension was assessed for each of the five time points studied. This was due to the difficulty of maintaining slides at $4{ }^{\circ} \mathrm{C}$ while assessing germination microscopically. Three isolates were included for each of the four clonal lineages studied (US-8, US-22, US-23, and US-24). Percentage of total germination that was indirect was calculated for each of the time points considered. The experiment was conducted at least twice for each isolate.

Effects of time and lineage on indirect germination were analyzed using JMP 9.0.2 (SAS Institute). A standard least square analysis was used, where replications were considered random terms, while time and lineage were considered as fixed effects. To determine whether mean germination, within a lineage, differed between time periods studied, a Tukey-Kramer HSD test with $\alpha=$ 0.05 was performed. To study differences in the rate at which indirect germination occurred over time (from 0 to $240 \mathrm{~min}$ after exposure of sporangia to 4 or $15^{\circ} \mathrm{C}$ ), data were transformed to achieve linearity. Data were adequately described by a negative exponential model (18) for all lineages studied. Slopes representing the rate of indirect germination over time were compared using a $t$ test with $\alpha$ $=0.05$.

\section{Results}

Isolates and isolation. In 2009, 2010, and 2011, approximately 350 samples were received and processed. In 2009, approximately 71 samples were received from six states (Florida, Maine, New Jersey, New York, Tennessee, and Virginia) within the United States, and 57 of these were isolated into culture and characterized. In 2010, approximately 81 samples were received from 14 states within the United States (Connecticut, Hawaii, Idaho, Kentucky, Louisiana, Massachusetts, Maryland, Maine, Montana, New Hampshire, New York, Pennsylvania, Washington, and Wisconsin) and one province of Canada (Ontario). Among these, 69 were successfully isolated into culture and characterized. In 2011, approximately 204 samples were received from 17 states within the United 
States (California, Connecticut, Delaware, Florida, Idaho, Maine, Minnesota, North Dakota, New Hampshire, New York, Ohio, Oregon, Pennsylvania, Rhode Island, Virginia, Washington, and Wisconsin) and one province of Canada (Ontario). Among these, 116 were successfully isolated and characterized. Isolation was successful on both pea and rye B agar, with rapid growth observed on pea agar.

Clonal lineages were defined according to mating type, mitochondrial haplotype, Gpi genotype, RG-57 DNA fingerprint profile (Table 3), and microsatellite genotype (Table 4). Clonal lineages US- 8 and US-22 were A2 mating type, whereas clonal lineages US-23 and US-24 were A1 mating type. All four clonal lineages were determined to have the Ia mitochondrial haplotype. Each lineage was described by a unique genotype at the locus for GPI. US-8 was $100 / 111 / 122$, US-22 was $100 / 122$, US-23 was $100 / 100$, and US-24 was 100/111 (Table 3). Each lineage showed a unique RFLP fingerprint as determined by RG57, with 10 bands being polymorphic for these four lineages (Table 3). Alleles at $11 \mathrm{mi}-$ crosatellite loci successfully distinguished the four clonal lineages studied (Table 4). Furthermore, for the isolates studied, two variants were identified within US-22 based on microsatellite results. These variants differed from the typical US-22 at markers Pi89 (Var1, eight isolates) and D13 (Var2, one isolate). Similarly, one variant was identified within US-24 at marker Pi02 (Table 4).

The predominant clonal lineages sampled were US-8, US-22, US-23, and US-24. Although US-8 is a well-characterized lineage, it was included in the study for comparative purposes. In 2009, only US-8 and US-22 were identified, with a total of 11 US-8 and 43 US-22 isolates. In 2010 and 2011, in total, 11 and 3 US-8, 33 and 25 US-22, 6 and 61 US-23, and 9 and 50 US-24 isolates, respectively, were collected for each year. The summary data for host of origin for all 3 years for each clonal lineage were as follows $(\mathrm{P}=$ potato and $\mathrm{T}=$ tomato $): \mathrm{US}-8,25(\mathrm{P})$ and $0(\mathrm{~T}) ; \mathrm{US}-22,15(\mathrm{P})$ and $86(\mathrm{~T})$; US-23, $13(\mathrm{P})$ and $54(\mathrm{~T})$; and US-24, $57(\mathrm{P})$ and $2(\mathrm{~T})$.

Sensitivity to mefenoxam. Sensitivity to mefenoxam was assessed for 206 isolates. In general, US-8 was resistant, US-22 and US-23 were sensitive, and US-24 was generally sensitive (Fig. 1). In all, $8,97,94$, and $75 \%$ of the isolates belonging to clonal lineages US-8, US-22, US-23 and US-24, respectively, grew less than $40 \%$ relative to the control $\left(0 \mu \mathrm{g} \mathrm{ml}^{-1}\right)$ on mefenoxam-amended plates ( 5 and $100 \mu \mathrm{g} \mathrm{ml}^{-1}$ ). Differences in mefenoxam sensitivity between years for each clonal lineage were analyzed using a standard least square model. For US-8, US-22, and US-23, mefenoxam sensitivity did not vary significantly among the years studied (US$8, P=0.36$; US-22, $P=0.36$; and US-23, $P=0.39$; Fig. 2 ). However, for US-24, isolates collected in 2011 were less sensitive to mefenoxam than those collected in 2010 ( $P=0.005$; Fig. 2$)$.

Due to the diversity in microsatellite genotype for the US-22 lineage (one dominant genotype and two variants), we tested whether there was diversity in mefenoxam sensitivity associated with either variant. US-22 variants at Pi89 (Var1, eight isolates) were only collected in 2010 whereas diversity at marker D13 (Var2, one isolate) was only found in 2011. US-22 Var1 did not differ significantly in its sensitivity to mefenoxam from the typical US-22 isolates collected and analyzed from $2010(P=0.75)$. Similarly, Var2, which was represented by a single isolate, did not differ significantly in its sensitivity to mefenoxam relative to the typical US-22 isolates analyzed from $2011(P=0.54)$.

Potato and tomato pathogenicity. The relative pathogenicity of the four clonal lineages on potato and tomato was assessed by measuring lesion size and sporulation at 6 days post inoculation. Lesion area ranged from $1.4 \mathrm{~cm}^{2}$ (US-24, on tomato) to $7.9 \mathrm{~cm}^{2}$

Table 3. Summary of multilocus genotypes of four Phytophthora infestans clonal lineages in the United States and Canada ${ }^{\mathrm{a}}$

\begin{tabular}{|c|c|c|c|c|c|c|c|c|c|c|c|c|c|c|c|c|c|c|c|c|c|c|c|c|c|c|c|c|c|}
\hline \multirow[b]{2}{*}{ Lineage } & \multirow[b]{2}{*}{ MT } & \multirow[b]{2}{*}{ Hap } & \multirow[b]{2}{*}{ GPI } & \multicolumn{26}{|c|}{ RG57 } \\
\hline & & & & 1 & 2 & 3 & 4 & 5 & 6 & 7 & 8 & 9 & 10 & 11 & 12 & 13 & 14 & 15 & 16 & 17 & 18 & 19 & 20 & 21 & 22 & 23 & 24 & $24 a$ & 25 \\
\hline US-8 & $\mathrm{A} 2$ & la & $100 / 111 / 122$ & 1 & 0 & 0 & 1 & 1 & 0 & 0 & 0 & 0 & 1 & 0 & 0 & 1 & 1 & 0 & 1 & 0 & 0 & 0 & 1 & 1 & 0 & 1 & 1 & 0 & 1 \\
\hline US-22 & $\mathrm{A} 2$ & la & $100 / 122$ & 1 & 0 & 0 & 0 & 1 & 0 & 0 & 0 & 0 & 0 & 0 & 0 & 1 & 1 & 0 & 1 & 0 & 0 & 0 & 1 & 1 & 0 & 0 & 1 & 0 & 1 \\
\hline US-23 & A1 & la & $100 / 100$ & 1 & 1 & 0 & 0 & 1 & 1 & 0 & 0 & 0 & 1 & 0 & 0 & 1 & 1 & 0 & 0 & 1 & 0 & 0 & 1 & 1 & 0 & 0 & 1 & 1 & 1 \\
\hline US-24 & A1 & la & $100 / 111$ & 1 & 0 & 1 & 0 & 1 & 0 & 1 & 0 & 0 & 1 & 0 & 0 & 1 & 1 & 0 & 1 & 0 & 0 & 0 & 1 & 1 & 0 & 1 & 1 & 0 & 1 \\
\hline
\end{tabular}

${ }^{\text {a }}$ MT = mating type, Hap = mitochondrial haplotype, and GPI = glucose-6-phosphate isomerase.

Table 4. Observed allele sizes for four clonal lineages of Phytophthora infestans amplified with 11 microsatellite markers

\begin{tabular}{|c|c|c|c|c|c|c|c|}
\hline \multirow[b]{2}{*}{ SSR marker ${ }^{a}$} & \multicolumn{7}{|c|}{ Size (bp) for each lineage (isolate identity) } \\
\hline & $\begin{array}{c}\text { US8 } \\
\text { (US100028) }\end{array}$ & $\begin{array}{c}\text { US22 } \\
\text { (US090042) }\end{array}$ & $\begin{array}{c}\text { US22 var1 } \\
\text { (US100001) }\end{array}$ & $\begin{array}{c}\text { US22 var2 } \\
\text { (US110002) }\end{array}$ & $\begin{array}{c}\text { US23 } \\
\text { (BL 2009 P4) }\end{array}$ & $\begin{array}{c}\text { US24 } \\
\text { (ND822P) }\end{array}$ & $\begin{array}{r}\text { US24 var1 } \\
\text { (US110159) }\end{array}$ \\
\hline Pi02 & & & & & 162 & & 162 \\
\hline $\mathrm{Pi} 02$ & 162 & 162 & 162 & 162 & 164 & 162 & 164 \\
\hline $\mathrm{Pi} 02$ & 164 & 164 & 164 & 164 & 166 & 164 & 166 \\
\hline $\mathrm{Pi} 4 \mathrm{~B}$ & 213 & 213 & 213 & 213 & 213 & 217 & 217 \\
\hline Pi4B & 226 & 213 & 213 & 213 & 217 & 226 & 226 \\
\hline Pi89 & 179 & 177 & 177 & 177 & 179 & 177 & 177 \\
\hline Pi89 & 179 & 179 & 181 & 179 & 179 & 179 & 179 \\
\hline Pi04 & 166 & 166 & 166 & 166 & 170 & 166 & 166 \\
\hline Pi04 & 170 & 170 & 170 & 170 & 170 & 170 & 170 \\
\hline Pi56 & 255 & 255 & 255 & 255 & 253 & 255 & 255 \\
\hline Pi56 & 255 & 255 & 255 & 255 & 255 & 255 & 255 \\
\hline Pi70 & 190 & 190 & 190 & 190 & 190 & 190 & 190 \\
\hline Pi70 & 190 & 193 & 193 & 193 & 190 & 193 & 193 \\
\hline PiG11 & 155 & 131 & 131 & 131 & 140 & 155 & 155 \\
\hline PiG11 & 155 & 155 & 155 & 155 & 155 & 155 & 155 \\
\hline Pi63 & 279 & 279 & 279 & 279 & 270 & 279 & 279 \\
\hline Pi63 & 279 & 279 & 279 & 279 & 279 & 279 & 279 \\
\hline D13 & 106 & Null & Null & 147 & 134 & 106 & 106 \\
\hline D13 & 110 & Null & Null & 147 & 134 & 110 & 110 \\
\hline Pi16 & 173 & 177 & 177 & 177 & 177 & 173 & 173 \\
\hline PI16 & 177 & 177 & 177 & 177 & 177 & 177 & 177 \\
\hline Pi33 & 203 & 203 & 203 & 203 & 203 & 203 & 203 \\
\hline Pi33 & 203 & 206 & 206 & 206 & 206 & 206 & 206 \\
\hline
\end{tabular}

${ }^{a}$ SSR $=$ simple-sequence repeat. 
(US-8, potato) (Fig. 3). US-8 and US-24 produced larger lesions on potato than on tomato $(P<0.0001)$. Although both US-22 and US-23 had slightly larger mean lesion areas on tomato compared with potato, these differences were not significant $(P=0.17$ and $P$ $=0.25$, respectively). For each of the clonal lineages studied, sporulation differed significantly between hosts $(P<0.0001$; Fig. 3 ). Mean sporulation ranged from 341 sporangia $\mathrm{ml}^{-1}$ (US-24 on tomato) to 16,308 sporangia $\mathrm{ml}^{-1}$ (US-23 on tomato). US-8 and US-24 sporulated more profusely on potato than on tomato, with a mean sporulation of 7,244 and 2,950 sporangia $\mathrm{ml}^{-1}$ on potato leaflets, respectively, versus 1,158 and 341 sporangia $\mathrm{ml}^{-1}$ on tomato, respectively. In contrast, lineages US-22 and US-23 sporulated more profusely on tomato leaflets, with a mean sporulation of 2,598 and 5,476 sporangia $\mathrm{ml}^{-1}$ on potato leaflets, respectively, versus 11,669 and 16,308 sporangia $\mathrm{ml}^{-1}$ on tomato, respectively.
When comparing among lineages, we found that US-22 and US-23 are pathogenic to both potato and tomato (Fig. 3). However, it seems that US-23 might be even more aggressive than US-22 on both potato and tomato. US-24 is pathogenic mainly on potato and not at all aggressive to tomato. These pathogenicity characteristics are similar to those of US-8 (Fig. 3).

To determine whether Var1 isolates (eight isolates total) within the US-22 lineage differed in host preference when compared with the typical US-22 isolates, these were analyzed as separate groups. For lesion area, no significant interaction was observed between the two US-22 genotypes and hosts $(P=0.47)$. For both genotypes, there may have been a trend toward larger lesion area on tomato than potato $(P=0.09)$. However, Var1 isolates produced smaller lesions on both potato and tomato than did the predominant US-22 isolates $(P=0.04)$. For Var1 isolates, lesion areas on potato and
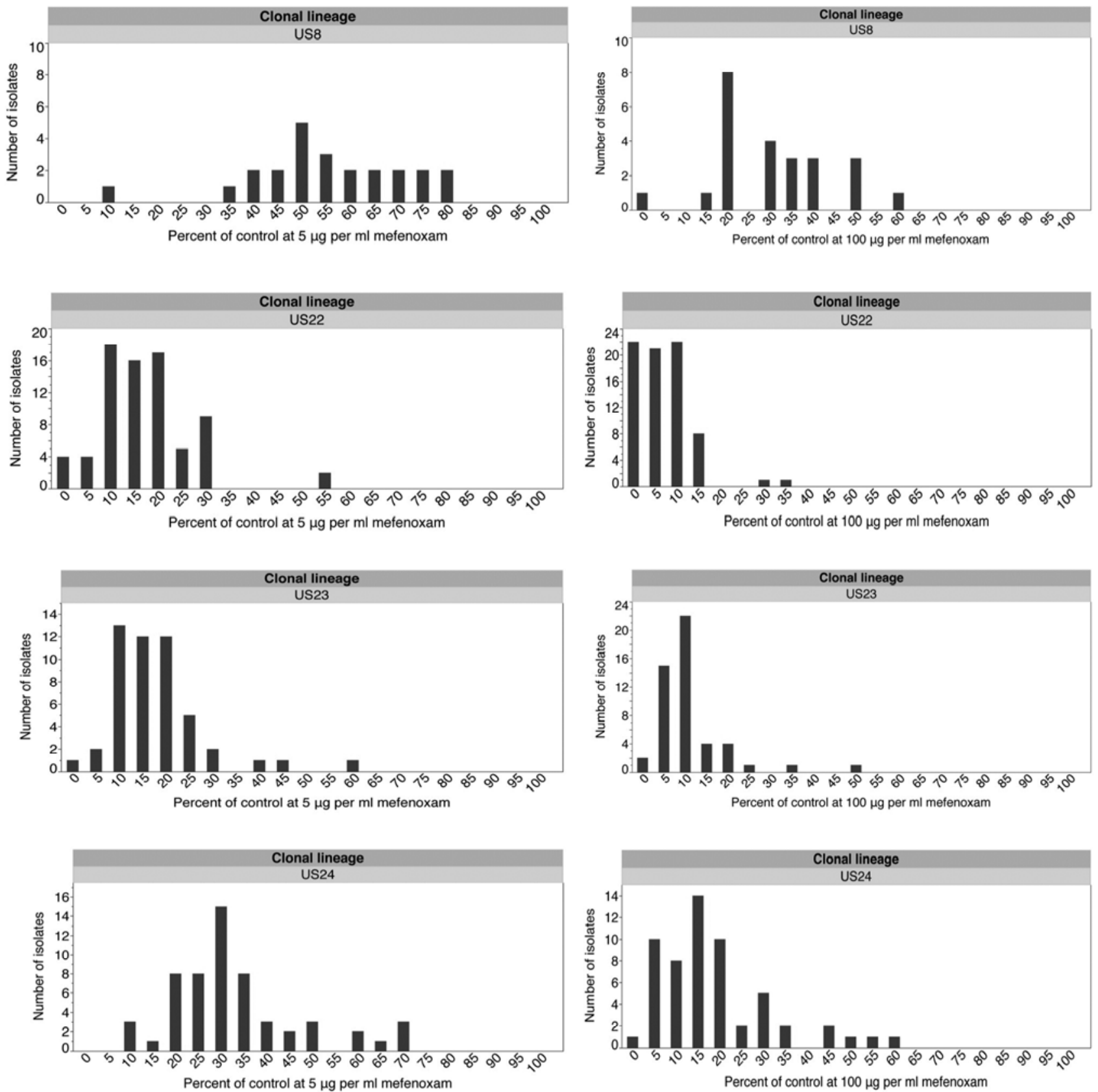

Fig. 1. Response of Phytophthora infestans isolates to mefenoxam. Relative growth (as percentage of control) at $5 \mu \mathrm{g} \mathrm{ml} \mathrm{l}^{-1}$ (left) and $100 \mu \mathrm{g} \mathrm{ml}{ }^{-1}$ (right) relative to control ( 0 $\mu \mathrm{g} \mathrm{ml}^{-1}$ ). Sample sizes for each lineage are US-8, $n=24$; US-22, $n=75$; US-23, $n=50$; and US-24, $n=57$, for a total sample size of 206 isolates. US-8 has been described as resistant given that $92 \%$ of the isolates grew more than $40 \%$ relative to the control $\left(0 \mu \mathrm{g} \mathrm{ml}^{-1}\right)$ on mefenoxam-amended plates $\left(5 \mathrm{and}^{100 \mu \mathrm{g} \mathrm{ml}} \mathrm{l}^{-1}\right)$, US-22 and US-23 have been described as sensitive given that 97 and $94 \%$ of the isolates respectively grew less than $40 \%$ relative to the control, and US- 24 has been described as generally sensitive given that $75 \%$ of the isolates grew less than $40 \%$ relative to the control. 
tomato were 4.98 and $5.40 \mathrm{~cm}^{2}$, respectively, and, for typical US22 isolates, lesion areas on potato and tomato were 5.55 and 6.58 $\mathrm{cm}^{2}$, respectively. For mean sporulation, the interaction between pathogen genotype (US-22 Var1 and the typical US-22 genotype) and host was significant $(P=0.01)$. This was due to reduced sporulation of Var1 on tomato compared with the typical US-22 $(P=$ 0.002 ). Sporulation on potato and tomato was 2,577 and 8,234 sporangia $\mathrm{ml}^{-1}$, respectively, for Var1 and 2,602 and 12,345 sporangia $\mathrm{ml}^{-1}$, respectively, for the typical US-22 isolates.

Temperature effect on total sporangial germination. The influence of temperature on sporangial germination was tested. In one experiment, germination was evaluated after $16 \mathrm{~h}$ at 10, 15, 20, and $25^{\circ} \mathrm{C}$ (Fig. 4) and, in a second experiment, germination was assessed after $16 \mathrm{~h}$ at 5 and $10^{\circ} \mathrm{C}$. Indirect germination was highest at 5 and $10^{\circ} \mathrm{C}$, with germination decreasing as temperature in-

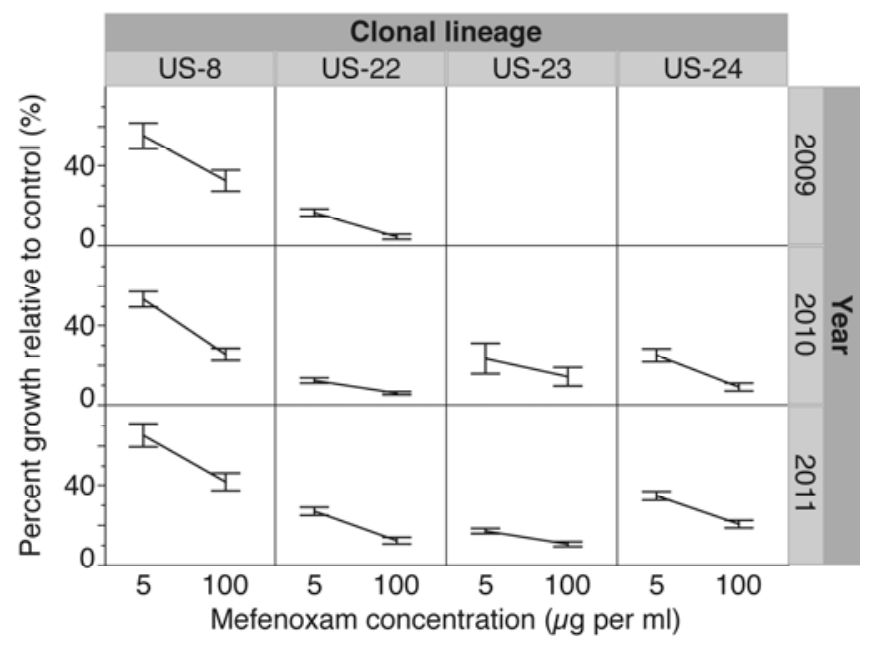

Fig. 2. Sensitivity to mefenoxam of four Phytophthora infestans clonal lineages in three consecutive years. Data represent the relative (radial) growth of isolates on medium containing mefenoxam at 5 and $100 \mathrm{\mu g} \mathrm{ml}^{-1}$ relative to growth with no mefenoxam. The number of isolates in the clonal lineages for 2009, 2010, and 2011 were US-8, $n=10,11$, and 3; US-22, $n=25,32$, and 18; US-23, $n=0,6$, and 44; and US-24, $n=0,9$, and 48, respectively, for a total sample size of 206 isolates. Error bars represent one standard error from the mean. creased to $20^{\circ} \mathrm{C}$. There were no significant differences in germination between temperatures 20 and $25^{\circ} \mathrm{C}$. Direct germination was not commonly observed but, when it occurred, it was mostly observed at higher temperatures $\left(20\right.$ and $25^{\circ} \mathrm{C}$; Fig. 4). In these two experiments, US- 8 and US-23 showed greater direct germination in comparison with US-22 and US-24 $(P<0.01)$. The isolates in US22 Var1 did not differ significantly from the typical US-22 genotype in either indirect or direct germination at any temperature. Differences in indirect germination between US-8 and US-24 at $10^{\circ} \mathrm{C}$ were significant $(P<0.0262)$. Overall, US-8 displayed the lowest indirect germination at $10^{\circ} \mathrm{C}$.

Effect of temperature on the rate of sporangial germination. The rate at which sporangia released zoospores was affected strongly by temperature and there were some large differences among clonal lineages (Fig. 5). Germination was much faster at 4 than at $15^{\circ} \mathrm{C}$. For example, within $90 \mathrm{~min}$, more than $30 \%$ of the isolates had released zoospores at $4^{\circ} \mathrm{C}$ whereas, at $15^{\circ} \mathrm{C}$, less than $20 \%$ of the isolates had released their zoospores. Sporangia of US24 released zoospores more rapidly than did sporangia of the other lineages at either 4 or $15^{\circ} \mathrm{C}$ (Fig. 5). For example, within $30 \mathrm{~min}$ at $4^{\circ} \mathrm{C}$, more than $75 \%$ of the US-24 sporangia had liberated zoospores, whereas zoospore release from the other lineages ranged from about 20 to about $35 \%$ (Fig. $5 \mathrm{~A}$ ). At $15^{\circ} \mathrm{C}$, zoospore release was slower for all lineages but, still, US-24 released zoospores from more sporangia than did the other lineages $(P<0.05)$. After $30 \mathrm{~min}$ at $15^{\circ} \mathrm{C}, 13 \%$ of the US-24 sporangia had released zoospores but only $4 \%$ of the US-23 sporangia had released zoospores (Fig. 5B).

\section{Discussion}

Several important phenotypic characteristics of the four dominant clonal lineages of $P$. infestans identified in samples from the 2009, 2010, and 2011 epidemics in the United States were determined. These lineages were initially defined on the basis of mating type, mitochondrial haplotype, Gpi genotype, and RG-57 DNA fingerprint profile but subsequently refined by the addition of microsatellite markers. The microsatellite markers identified some genotypic diversity within some clonal lineages.

The ability to categorize individuals into clonal lineages has been helpful for the study of migration patterns and population biology of $P$. infestans (7). Once the phenotype is known, it is
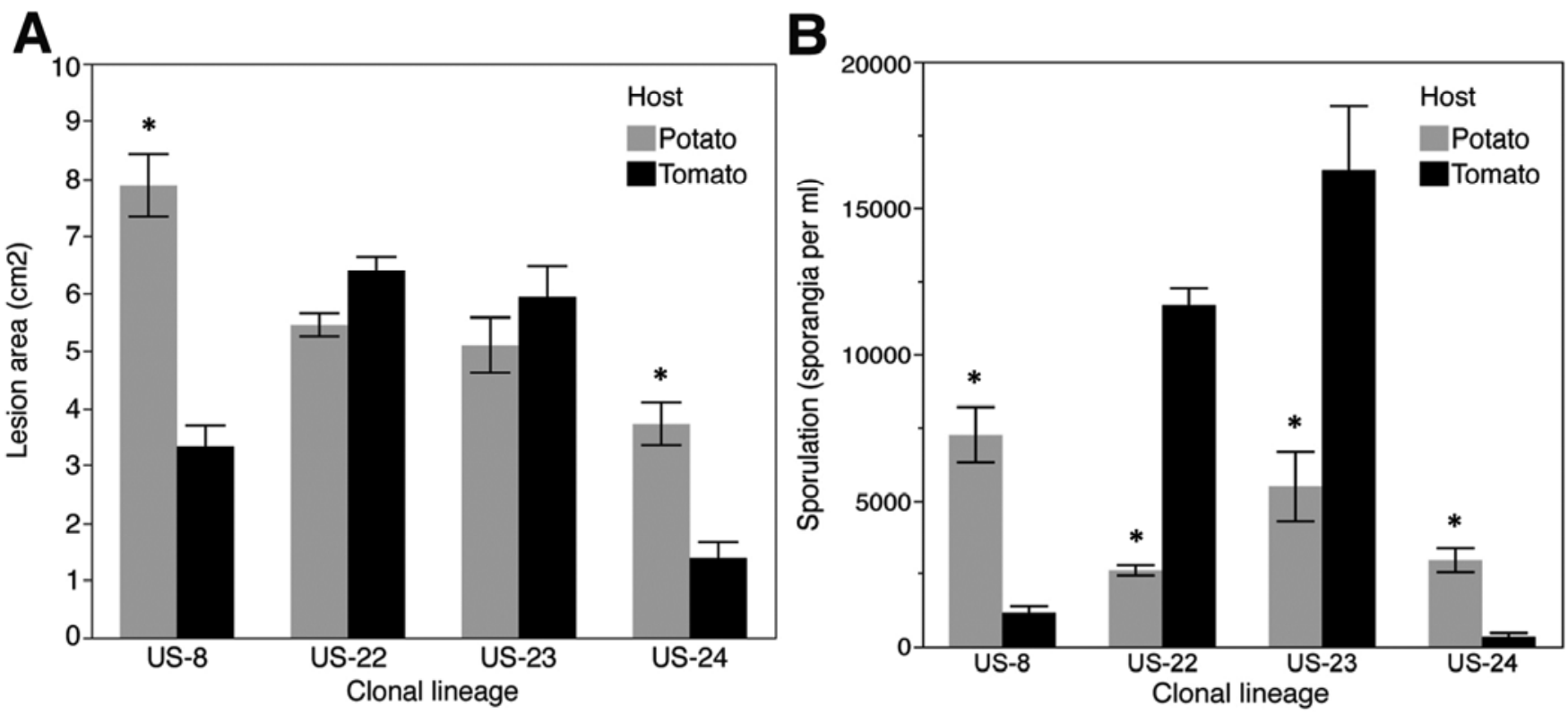

Fig. 3. Pathogenicity on potato and tomato for isolates within four clonal lineages of Phytophthora infestans. A, Lesion areas produced on potato and tomato. B, Sporangia produced per infection site on potato and tomato. Clonal lineages US-8, US-22, US-23, and US-24 include 8, 34, 7, and 9 isolates, respectively. Bars represent mean lesion area for $A$ and mean sporulation (per infection site) for B for all isolates within each clonal lineage. Error bars represent one standard error from the mean. The asterisk $\left(^{*}\right)$ indicates a significant difference within a clonal lineage for either sporulation or lesion size for potato versus tomato. Lesion areas and sporulation were measured at 6 days post inoculation. 
possible to make lineage-specific management recommendations. This study reports phenotypes for sensitivity to mefenoxam, host preference, and germination at different temperatures for the recently important clonal lineages in the United States (US-22, US23, and US-24).
Sensitivity to mefenoxam was tested in an in vitro assay comparing three different concentrations of the active ingredient $(0,5$, and $100 \mu \mathrm{g} \mathrm{ml}^{-1}$ ). The recently described clonal lineages (US-22, US-23, and US-24) were generally sensitive to mefenoxam (Fig. 1). US-8, which has been dominant on potato in the United States

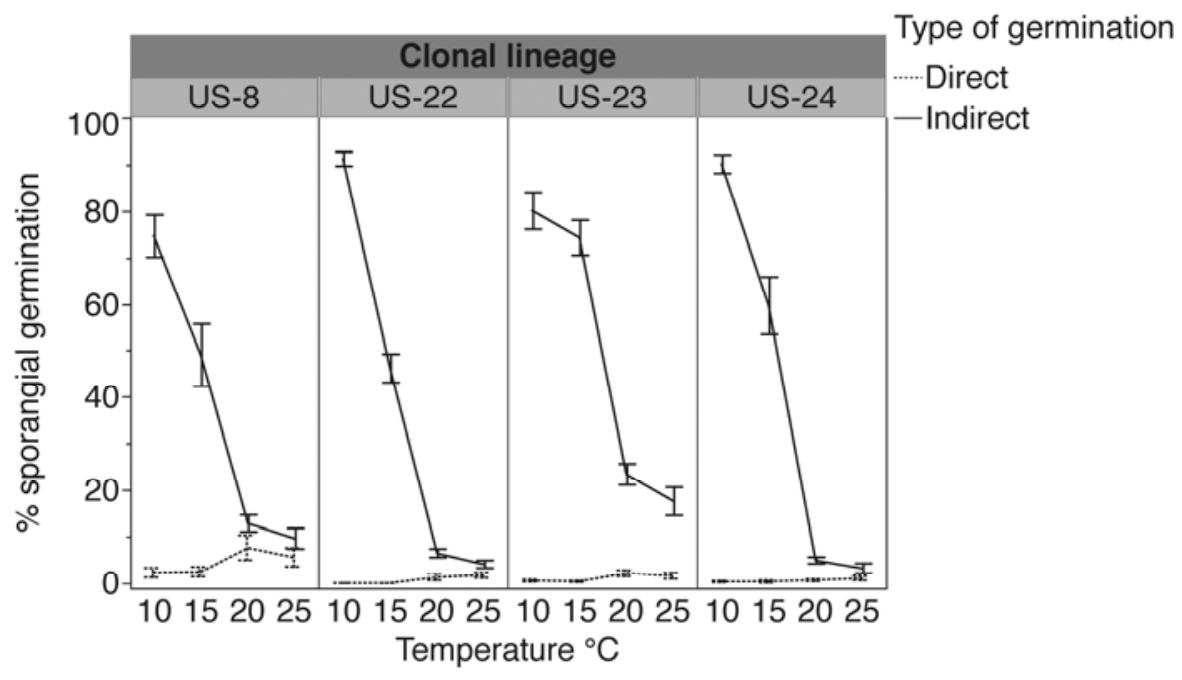

Fig. 4. Proportion of sporangia that had germinated within $16 \mathrm{~h}$ at $10,15,20$, and $25^{\circ} \mathrm{C}$. Data points indicate the mean percentage of germination of three isolates in three independent experiments. Error bars represent one standard error from the mean.
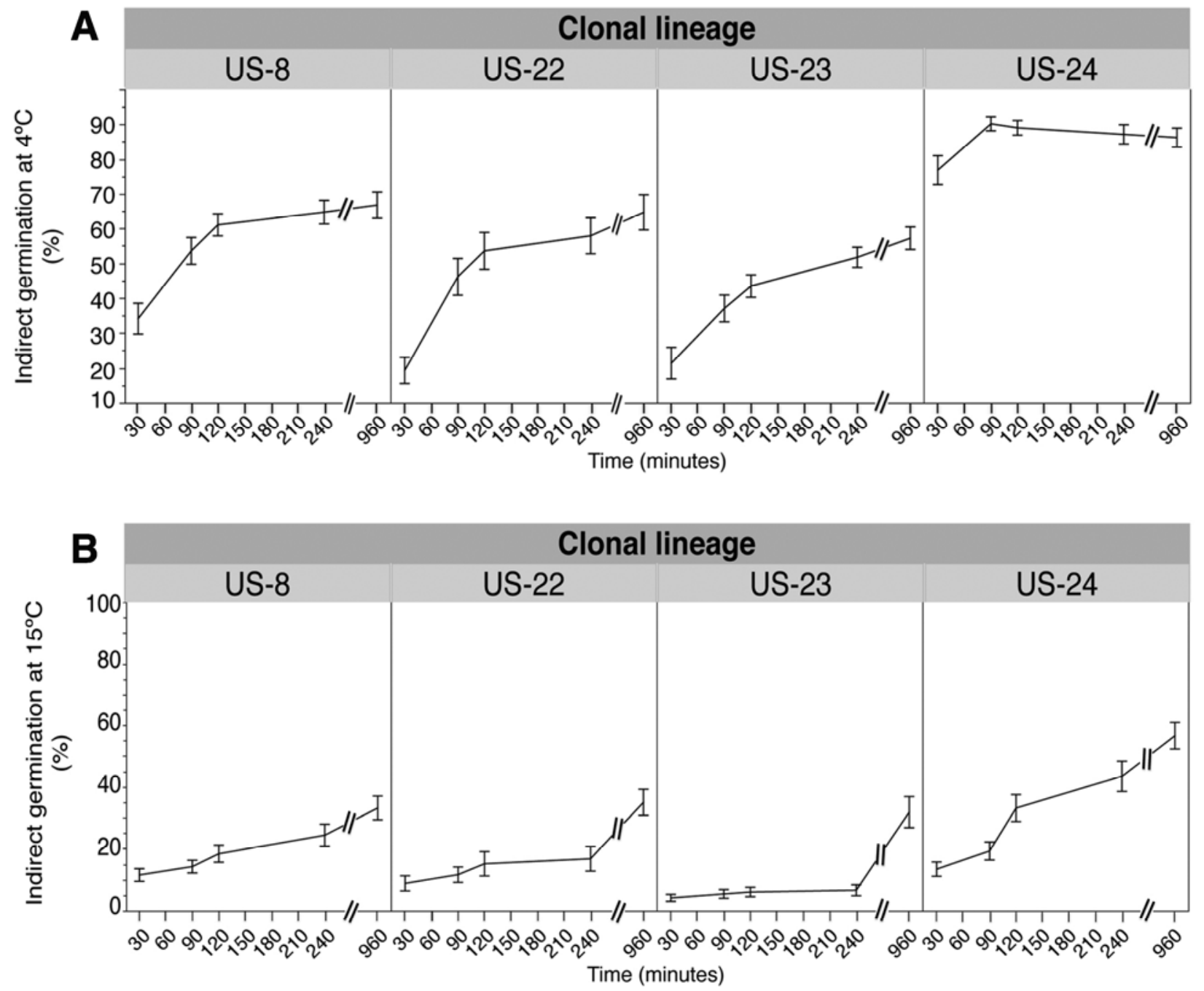

Fig. 5. Proportion of sporangia that had germinated at $30,90,120,240$, and 960 min at $A, 4^{\circ} \mathrm{C}$ or $\mathbf{B}, 15^{\circ} \mathrm{C}$. Data points are the means obtained for three isolates in three independent experiments. Error bars represent one standard error from the mean. 
since the mid-1990s (4) and is now being displaced by novel clonal lineages, has maintained stable resistance to mefenoxam. Notably, US-24 showed an increase in the mean colony growth on mefenoxam-amended plates for isolates from 2011 in comparison with isolates from 2010, indicating a potential decrease in sensitivity to mefenoxam. As far as we know, there has not been an increased use of mefenoxam; therefore, we do not know of a factor to explain the decreased sensitivity. This decrease in sensitivity might be the result of a new better-fit subpopulation of the pathogen, although a deeper analysis must be performed to confirm this hypothesis. Knowledge regarding the sensitivity of the lineages to mefenoxam is important in terms of selecting the most appropriate fungicide.

Host preference was studied on potato (Yukon Gold) and tomato (Rutgers). Clonal lineages US- 8 and US-24 showed a preference for potato as a host and were not at all aggressive on tomato. In contrast, US-22 and US-23 showed a preference for tomato although they were also pathogenic on potato. The original host from which these samples were collected reflected the same findings. In the years studied, $100 \%$ of US-8, $15 \%$ of US-22, $19 \%$ of US-23, and $97 \%$ of US-24 isolates were collected from potato. Differences observed among clonal lineages on Rutgers and Yukon Gold are consistent with the fact that US-24 and US-8 were typically reported on potato and not on tomato, whereas US-22 and US-23 have been typically reported on both potato and tomato.

The lineage-host combination determined the type of growth observed. US- 8 and US-24 caused large necrotic lesions and profuse sporulation on potato, whereas limited necrosis and very little sporulation was observed on tomato. Clonal lineages US-22 and US-23 caused necrotic lesions on potato and profuse biotrophic growth on tomato (Fig. 6). This biotrophic growth might have resulted in an underestimation of lesion area, given that it was difficult to define the boundaries of the lesions.

Eight members of the US-22 lineage (Var1) differed from others by one allele. This variant is apparently less fit on tomato. It was interesting to note that this SSR variation is likely to be a neutral change that allows tracking of a subclone. It is intriguing that the US-22 variant 2 changes from null to $147 \mathrm{bp}$ at the D13 locus. The chance of a back-mutation from null to amplification seems slim. However, the SSR analysis was performed at least twice for the variant individuals and is unlikely to be an error. We presently do not have a satisfactory model to explain this situation.

It has been known for nearly a century that late blight is highly dependent on environmental conditions $(19,23)$. When sporangia are deposited on a surface and then exposed to free moisture and cool temperatures, they may germinate indirectly by releasing between 8 and 12 biflagellated zoospores, each capable of initiating an infection (3). The effect of temperature on sporangial germi- nation has been extensively studied during the early 1900s by Melhus (19) and Crosier (2) and in the late 1900s by Mizubuti and Fry (21). Both Crosier (2) and Melhus (19) found that the optimal temperature for indirect germination was approximately $15^{\circ} \mathrm{C}$. Mizibuti and Fry (21) found similar results for isolates within clonal lineage US-1, suggesting that the isolates studied by Melhus and Crosier at the beginning of the 1900s may have belonged to the US-1 clonal lineage. In contrast to US-1, Mizubuti and Fry (21) found that isolates belonging to US-7 and US-8 lineages germinated indirectly better at lower temperatures (approximately $10^{\circ} \mathrm{C}$ ). These reports are in accordance with the results of the present study, where isolates belonging to clonal lineages US-8, US-22, US-23, and US-24 released zoospores better at 5 and $10^{\circ} \mathrm{C}$ than they did at higher temperatures. Similar to these previous studies, indirect germination at $20^{\circ} \mathrm{C}$ was reduced to less than half the maximum germination recorded. Direct germination was not commonly observed but, when it occurred, it was mostly observed at higher temperatures $\left(20\right.$ and $\left.25^{\circ} \mathrm{C}\right)$. These results are consistent with those reported by Melhus (19) and Crosier (2).

Indirect germination rate was also influenced by temperature. Lower temperatures $\left(4\right.$ compared with $15^{\circ} \mathrm{C}$ ) resulted in increased indirect germination rate and increased total germination. Differences in rate of indirect germination between clonal lineages were observed, with the most striking difference observed for clonal lineage US-24, where indirect germination was dramatically faster than that of any of the other three clonal lineages studied. As suggested by Mizubuti and Fry (21), rapid germination might enable the pathogen to overcome environmental limitations. The release of zoospores at lower temperatures could play an important role in disease development under conditions where low temperatures and short wet periods are frequent. Among the clonal lineages studied, US-24 might possess a competitive advantage under conditions of short, cool infectious periods. The differences in germination rate and response to temperature will be incorporated in the late-blight decision support model.

If sexual reproduction continues to remain limited in the United States and Canada, populations of the pathogen in these regions will remain relatively simple and, perhaps, stable. An understanding of phenotypic traits associated with unique clonal lineages of $P$. infestans has direct implications for potato and tomato late blight management. With the availability of rapid genotype analyses and rapid communication, in-season disease management may be adjusted based on the results of rapid genotypic analyses.

\section{Acknowledgments}

This work was supported by Cornell University and by the Agriculture and Food Research Initiative Competitive Grants Program Grant 2011-68004-30154
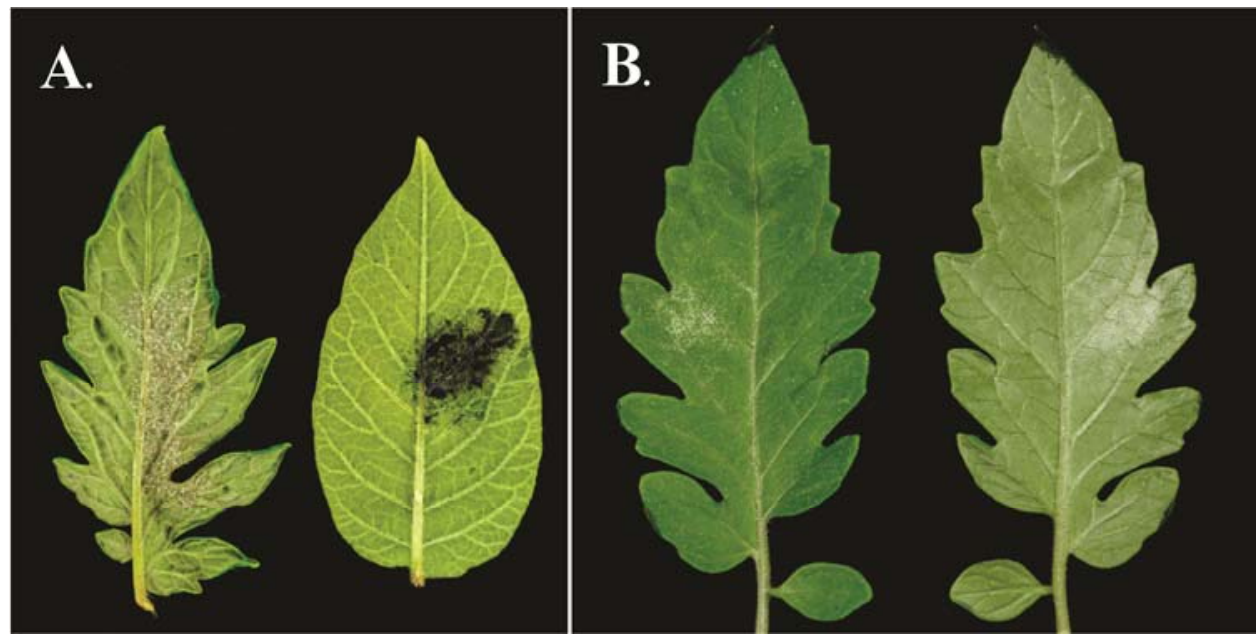

Fig. 6. Biotrophic growth was observed on tomato leaflets 6 days after inoculation and incubation at $15^{\circ} \mathrm{C}$. A, Growth of an isolate of Phytophthora infestans belonging to clonal lineage US-22 on tomato (left) and on potato (right). B, Growth of an isolate of $P$. infestans belonging to clonal lineage US-23 on the adaxial side of a tomato leaflet (left) and on the abaxial side of the same leaflet (right). 
from the USDA, and by the USDA NE-IPM Regional IPM program Special Grant NYC-153560. Thanks to the many people who sent diseased samples to the lab (Supplementary Table S1).

\section{Literature Cited}

1. Caten, C. E., and Jinks, J. L. 1968. Spontaneous variability of single isolates of Phytophthora infestans. I. Cultural variation. Can. J. Bot. 46:329-348.

2. Crosier, W. 1934. Studies in the biology of Phytophthora infestans (Mont.) De Bary. Cornell Univ. Exp. Stn. Mem. 155:1-40.

3. Erwin, D. C., and Ribeiro, O. K. 1996. Phytophthora Diseases Worldwide. American Phytopathological Society, St. Paul, MN.

4. Fry, W. E., and Goodwin, S. B. 1997. Re-emergence of potato and tomato late blight in the United States and Canada. Plant Dis. 81:1349-1357.

5. Goodwin, S. B., Cohen, B. A., and Fry, W. E. 1994. Panglobal distribution of a single clonal lineage of the Irish potato famine fungus. Proc. Natl. Acad. Sci. USA 91:11591-11595.

6. Goodwin, S. B., Schneider, R. E., and Fry, W. E. 1995. Cellulose-acetate electrophoresis provides rapid identification of allozyme genotypes of Phytophthora infestans. Plant Dis. 79:1181-1185.

7. Goodwin, S. B., Smart, C. D., Sandrock, R. W., Deahl, K. L., Punja, Z. K., and Fry, W. E. 1998. Genetic change within populations of Phytophthora infestans in the United States and Canada during 1994 to 1996: role of migration and recombination. Phytopathology 88:939-949.

8. Goodwin, S. B., Sujkowski, L. J., and Fry, W. E. 1995. Rapid evolution of pathogenicity within clonal lineages of potato late blight disease fungus. Phytopathology 85:669-676.

9. Goodwin, S. B., Sujkowski, L. S., and Fry, W. E. 1996. Widespread distribution and probable origin of resistance to metalaxyl in clonal genotypes of Phytophthora infestans in the United States and Western Canada. Phytopathology 86:793-799.

10. Goodwin, S. B. A., Drenth, A., and Fry, W. E. 1992. Cloning and genetic analyses of two highly polymorphic, moderately repetitive nuclear DNAs from Phytophthora infestans. Curr. Genet. 22:107-115.

11. Griffith, G. W., and Shaw, D. S. 1998. Polymorphisms in Phytophthora infestans: four mitochondrial haplotypes are detected after PCR amplification of DNA from pure cultures or from host lesions. App. Environ. Microbiol. 64:4007-4014.

12. Hu, C. H., Perez, F., Donohoo, R., McLeod, A., Myers, K., Ivors, K., Secor, G., Roberts, P., Deahl, K. L., Fry, W. E., and Ristaino, J. B. 2012. Recent genotypes of Phytophthora infestans in eastern USA reveal clonal populations and reappearance of mefenoxam sensitivity. Plant Dis. 96:1323-1330.

13. Jaime-Garcia, R., Trinidad-Correa, R., Felix-Gastelum, R., Orum, T. V., Wasmann, C. C., and Nelson, M. R. 2000. Temporal and spatial patterns of genetic structure of Phytophthora infestans from tomato and potato in the
Del Fuerte Valley. Phytopathology 90:1188-1195.

14. Knapova, G., and Gisi, U. 2002. Phenotypic and genotypic structure of Phytophthora infestans populations on potato and tomato in France and Switzerland. Plant Pathol. 51:641-653.

15. Lambert, D. H., and Currier, A. I. 1997. Differences in tuber rot development for North American clones of Phytophthora infestans. Am. Potato J. 74:39-43.

16. Lees, A. K., Wattier, R., Shaw, D. S., Sullivan, L., Williams, N. A., and Cooke, D. E. L. 2006. Novel microsatellite markers for the analysis of Phytophthora infestans populations. Plant Pathol. 55:311-319.

17. Legard, D. E., Lee, T. Y., and Fry, W. E. 1995. Pathogenic specialization in Phytophthora infestans: aggressiveness on tomato. Phytopathology 85:1362-1367.

18. Madden, L. V., Hughes, G., and van den Bosch, F. 2007. The Study of Plant Disease Epidemics. American Phytopathological Society, St. Paul, MN.

19. Melhus, I. E. 1915. Germination and infection with the fungus of the late blight of potato (Phytophthora infestans). Agric. Exp. Stn. Univ. Wis. Res Bull. 37:1-64.

20. Miller, J. S., Johnson, D. A., and Hamm, P. B. 1998. Aggressiveness of isolates of Phytophthora infestans from the Columbia Basin of Washington and Oregon. Phytopathology 88:190-197.

21. Mizubuti, E. S. G., and Fry, W. E. 1998. Temperature effects on developmental stages of isolates of three clonal lineages of Phytophthora infestans. Phytopathology 88:837-843.

22. Myers, K., Small, I., Jensen, S., Zuluaga, P., Guha Roy, S., and Fry, W. 2010. Characterization of Phytophthora infestans isolates from potato/tomato in 2010. Northeastern Division Meeting of the American Phytopathological Society.

23. Sato, N. 1994. Effect of sporulating temperature on the limit temperature in indirect germination of the sporangia of Phytophthora infestans. Ann. Phytopathol. Soc. Jpn. 60:60-65.

24. Small, I. M., Myers, K., Danies, G., Guha Roy, S., Bekoscke, K., and Fry, W. 2012. Characterization of recent clonal lineages of Phytophthora infestans in the United States using microsatellite markers. Annual Meeting of the American Phytopathological Society.

25. Spielman, 1. J., Drenth, A., Davidse, L. C., Sujkowski, L. J., Gu, W., Tooley, P. W., and Fry, W. E. 1991. A second world-wide migration and population displacement of Phytophthora infestans? Plant Pathol. 40:422-430.

26. Stevens, N. E. 1933. The dark ages in plant pathology in America: 1830 1870. J. Wash. Acad. Sci. 23:435-446.

27. Therrien, C. D., Tooley, P. W., Spielman, 1. J., Fry, W. E., Ritch, D. L., and Shelly, S. E. 1993. Nuclear DNA content, allozyme phenotypes and metalaxyl sensitivity of Phytophthora infestans from Japan. Mycol. Res. 97:945-950. 